Electrical characterization of nanocrystalline Si films by scanning tunnelling spectroscopy and beam-induced current in the scanning tunnelling microscope

This article has been downloaded from IOPscience. Please scroll down to see the full text article.

2003 Nanotechnology 1465

(http://iopscience.iop.org/0957-4484/14/1/315)

View the table of contents for this issue, or go to the journal homepage for more

Download details:

IP Address: 147.96.14.16

The article was downloaded on 21/02/2013 at 13:32

Please note that terms and conditions apply. 


\title{
Electrical characterization of nanocrystalline Si films by scanning tunnelling spectroscopy and beam-induced current in the scanning tunnelling microscope
}

\author{
E Nogales, B Méndez, J Piqueras and R Plugaru \\ Departamento de Física de Materiales, Facultad de Ciencias Físicas, Universidad Complutense \\ de Madrid, 28040, Madrid, Spain
}

Received 9 September 2002

Published 16 December 2002

Online at stacks.iop.org/Nano/14/65

\begin{abstract}
Nanocrystalline silicon films, with an average nanocrystal size of about $10 \mathrm{~nm}$, obtained by boron implantation of amorphous silicon layers, have been studied by remote-beam-induced current (REBIC) in a scanning tunnelling microscope (STM) and by current imaging tunnelling spectroscopy. STM images reveal a cell structure with cell sizes of about $200 \mathrm{~nm}$. STM-REBIC images display space-charge regions associated with the cell boundaries. The STM-REBIC contrast has been found to depend on the implantation dose and the thermal treatment given to the sample. The results show the capability of STM-REBIC to image electrically active regions in nanocrystalline silicon films with a resolution of up to $10 \mathrm{~nm}$.
\end{abstract}

\section{Introduction}

The study of the physical properties of nanocrystalline silicon (nc-Si) has attracted much attention in recent years because of its potential application in optoelectronic devices. The structure and properties of different forms of nc-Si such as those formed by $\mathrm{Si}$ ion implantation into an oxide layer, e.g. [1], nc-Si films prepared by crystallization of amorphous silicon [2] or porous silicon [3], have been widely investigated. The luminescence in the visible range of nc$\mathrm{Si}$ is basically explained in the framework of a quantum confinement model [3], but the influence of electronic states at the surface of nanocrystals or at the interface between nanocrystals and oxides or amorphous phases has to be considered in order to interpret the results $[4,5]$. For this reason electronic characterization of the structural features present in the nanocrystalline films is of interest.

Beam-induced modes in the scanning electron microscope (SEM) such as cathodoluminescence (CL), electron-beaminduced current (EBIC) or the EBIC technique known as remote EBIC (REBIC) have been widely applied in the characterization of several semiconductors. In particular,
REBIC was used to study the local electronic structure of grain boundaries in electroceramics [6] and silicon [7]. However, since the spatial resolution of SEM techniques is typically in the micron-submicron range, the use of scanning tunnelling microscopes (STMs) and related techniques is necessary to achieve resolution in the nanometre range. This is the case of the STM analogous to the SEM-REBIC which has been previously employed to image electrically active grain boundaries in $\mathrm{ZnO}$ with a high spatial resolution [8]. In the present work, STM-REBIC and current imaging tunnelling spectroscopy (CITS) measurements were used to characterize nc-Si films.

\section{Experimental method}

Silicon polycrystalline films, with a thickness of about $2 \mu \mathrm{m}$ were obtained by low-pressure chemical vapour deposition (LPCVD) on p-type silicon wafers at a temperature of $570{ }^{\circ} \mathrm{C}$ and a pressure of 0.4 Torr. The films were implanted with boron ions with an energy of $100 \mathrm{keV}$ and a dose in the range $10^{13}-10^{15} \mathrm{~cm}^{-2}$. Some of the samples were annealed at $650^{\circ} \mathrm{C}$ for $1 \mathrm{~h}$. The microscopic measurements were performed in 

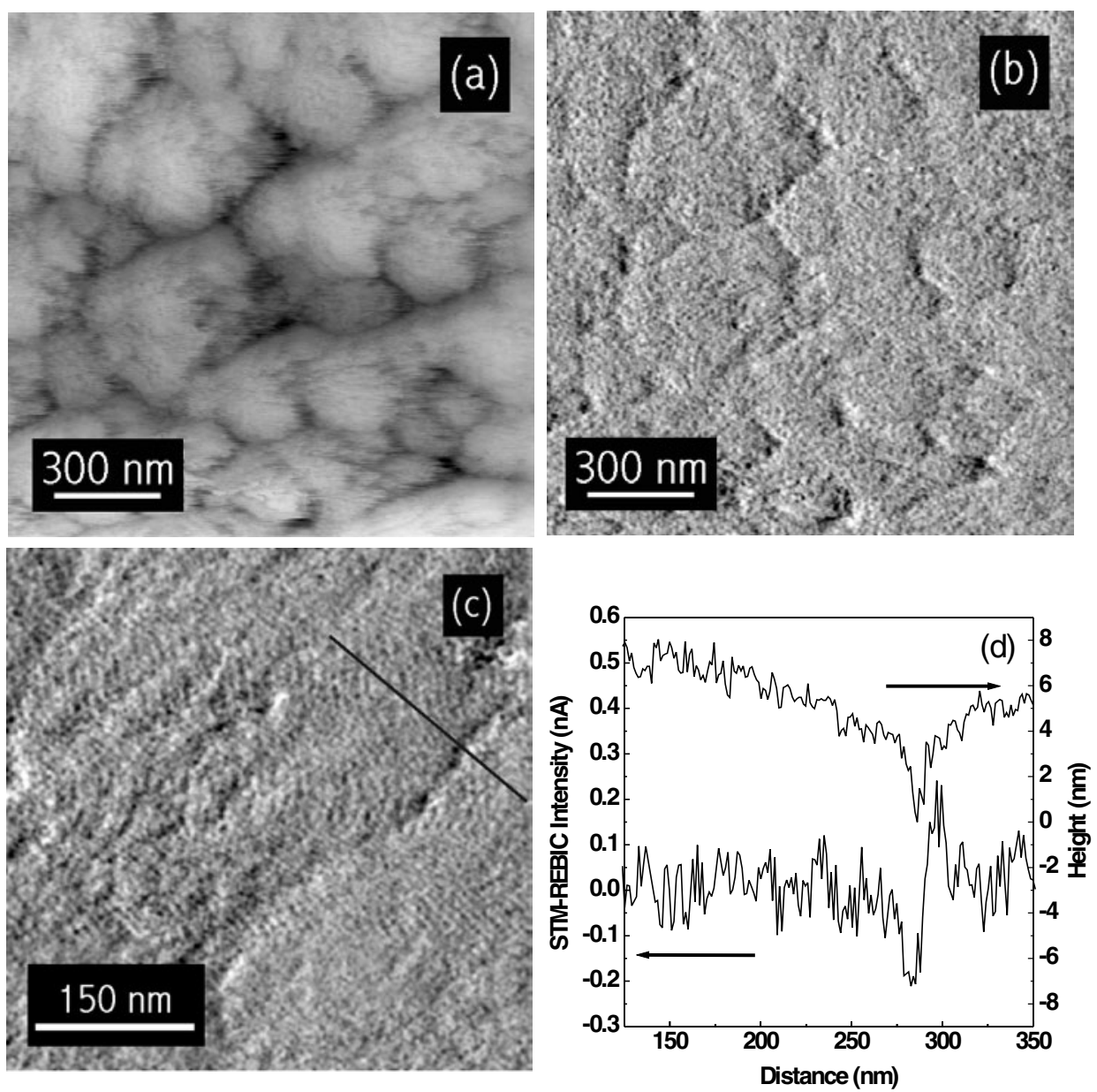

Figure 1. (a) STM constant current image of a-Si implanted with a boron dose of $10^{15}$ ions $\mathrm{cm}^{-2}$ acquired with a tip voltage of $3 \mathrm{~V}$ and $1.5 \mathrm{nA}$ tunnelling current. (b) Corresponding STM-REBIC image. (c) STM-REBIC image showing a detail of one of the boundaries. (d) Intensity profile of the marked line in (c).

a combined SEM-STM system based on a Leica 440 SEM operating under a vacuum of $1 \times 10^{-6}$ Torr. The small size of the STM enabled it to be mounted on the SEM specimen holder. Mechanically sharpened Pt-Ir wires were used as probe tips for tunnelling experiments. The STM was used in the conventional constant-current mode, in the current imaging tunnelling mode and in the STM-REBIC mode. CITS provides real space imaging of surface electronic states by recording $I-$ $V$ characteristic curves at fixed tip-sample separation at every pixel within an image [9, 10]. For the STM-REBIC mode, two ohmic contacts were provided by small HgIn dots on the sample surface connected to $\mathrm{Au}$ or PtIr wires. The tunnel tip was located on the region between the contacts and the current was measured at room temperature with a Keithley 428 current amplifier.

\section{Results and discussion}

The nanocrystalline films were formed by crystallization of the amorphous matrix during boron implantation and consisted of nanocrystals with an average size of about $10 \mathrm{~nm}$ arranged inside a cell structure as previously found by $\mathrm{x}$-ray diffraction and STM techniques $[11,12]$. The cell structure of the sample implanted with $10^{15}$ ions $\mathrm{cm}^{-2}$ is shown in the constant current
STM image of figure 1(a). The average size of cells was around $200 \mathrm{~nm}$. The STM-REBIC image of the same region is shown in figure 1(b) and reveals a contrast associated with the presence of space-charge regions at the cell boundaries. A detail of a boundary in the STM-REBIC mode and the corresponding line profile of the signal are shown in figures 1(c) and (d) respectively. It can be observed that the width of the line boundary revealed in the STM-REBIC image is about of $20 \mathrm{~nm}$. CITS images of the samples display a contrast associated with the cell boundaries (figure 2), which shows differences in the local electronic properties. For positive bias, boundaries appear darker than cells (figure 2(b)) while for negative bias the boundaries appear brighter (figure 2(c)). This result indicates a higher value of the surface energy band gap in the inner of the cell, which contains the nanocrystals, than at the boundary.

STM constant current images of the annealed samples reveal a similar cell structure to that in the untreated samples but no STM-REBIC contrast is detected. This result shows that the electrically active barriers between cells disappear during annealing and that the STM-REBIC contrast of the unannealed samples is related to electrically active regions and is not influenced by the topography of the sample. On the other hand, CITS images are also featureless in the range from +3.0 

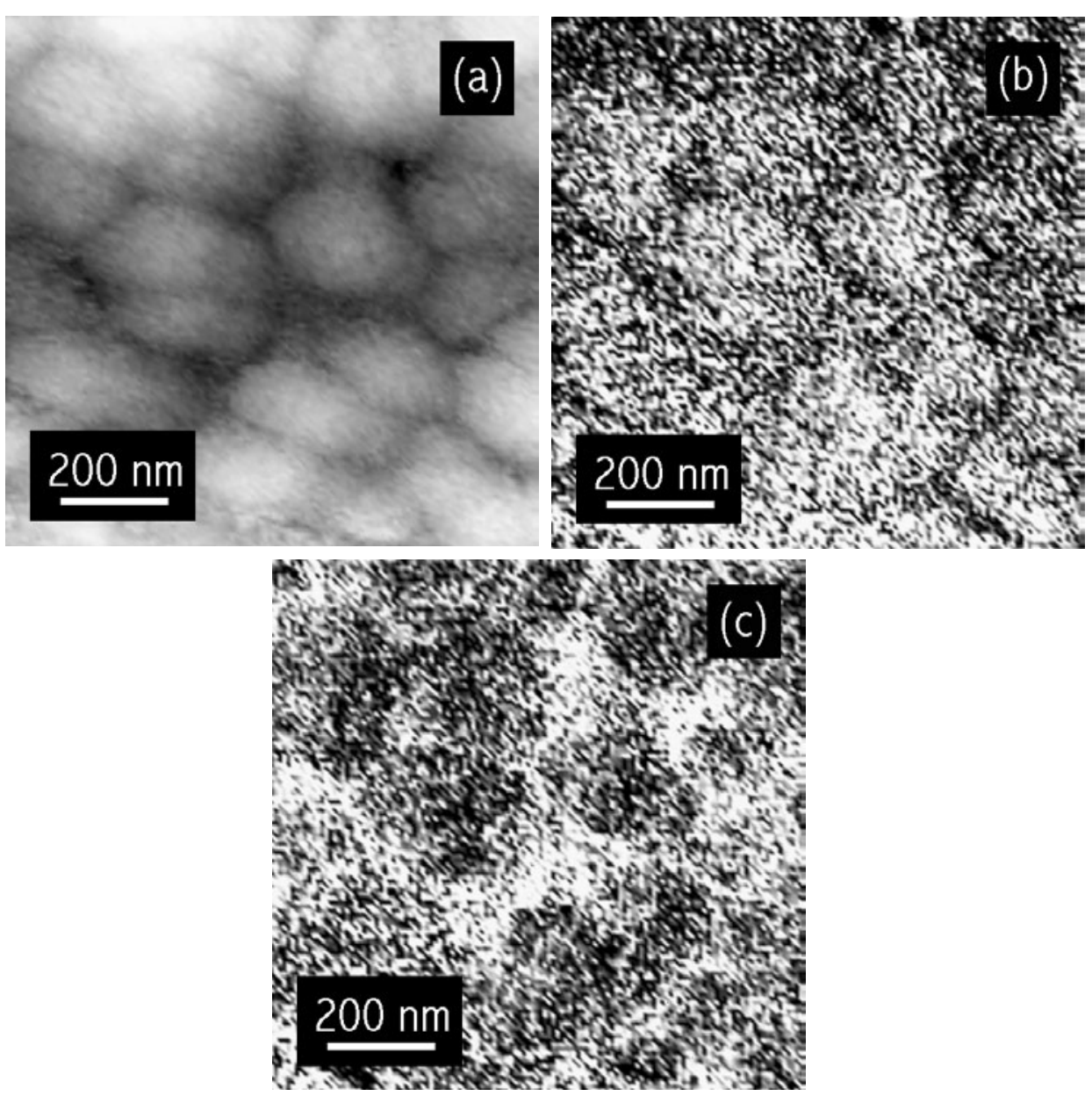

Figure 2. (a) STM constant current image of a-Si implanted with a boron dose of $10^{15}$ ions $\mathrm{cm}^{-2}$ acquired with a sample voltage of $-1.5 \mathrm{~V}$ and $0.4 \mathrm{nA}$ tunnelling current. (b), (c) Corresponding CITS images acquired at $3 \mathrm{~V}$ (b) and $-3 \mathrm{~V}$ (c) sample voltages.
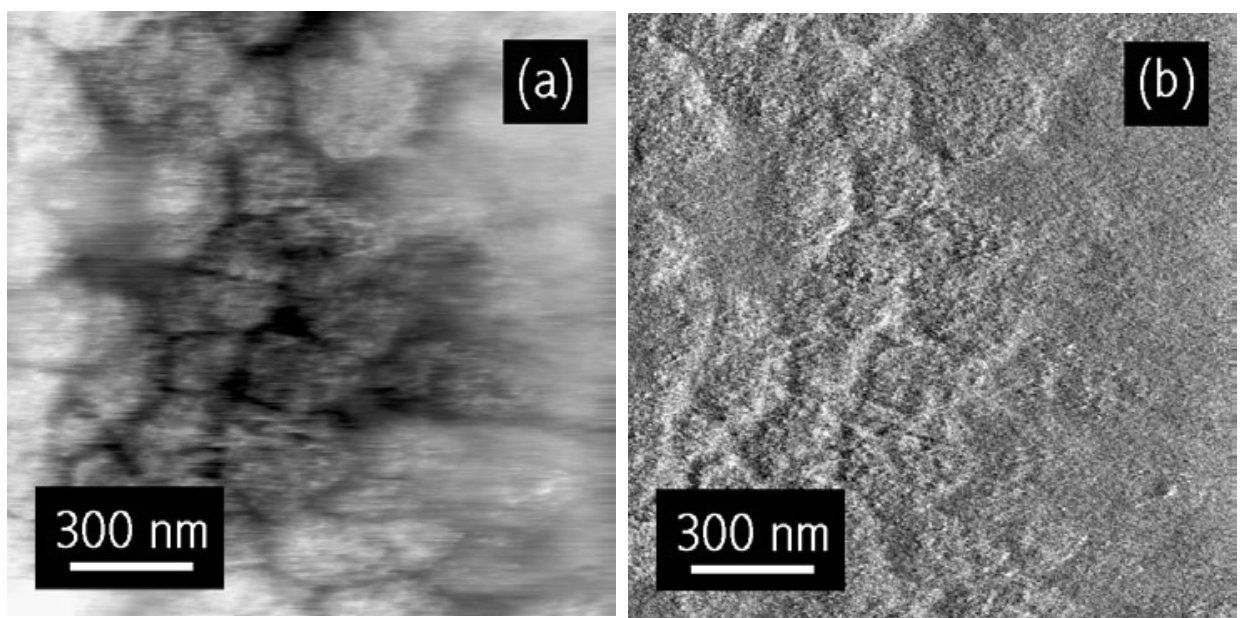

Figure 3. (a) STM constant current image of a-Si implanted with a boron dose of $10^{13}$ ions $\mathrm{cm}^{-2}$ acquired with a tip voltage of $-3.6 \mathrm{~V}$ and $1.8 \mathrm{nA}$ tunnelling current. (b) STM-REBIC image of the area shown in (a).

to $-3.0 \mathrm{~V}$ after annealing. This can be explained by the fact that thermal treatment induces an increase in the conductivity of the samples during the recrystallization process and a change of the defect structure and distribution at the cell boundaries.

In as-implanted a-Si with a lower dose of boron $\left(10^{13} \mathrm{ions}^{-2}\right)$ it was not possible to obtain a stable tunnelling current due to its high resistivity and therefore no STM images have been recorded. After annealing, tunnelling measurements could be performed and some cell boundaries were observed in the beam-induced images. A representative STM-REBIC image is shown in figure 3 , where the corresponding constant current image is also shown. The beam-induced signal was 
found to depend on the cell boundary considered and not all boundaries were revealed in the STM-REBIC images.

As stated above, the REBIC mode in the SEM has been successfully applied in the past to investigate electrically active defects in high-resistivity semiconductors while STMREBIC has been only occasionally used to image defects in materials such as $\mathrm{CuInSe}_{2}$ [13], diamond [14] or sintered zinc oxide [8]. The signal generation in SEM-REBIC is mainly due to the presence of space-charge regions and it provides direct evidence for electrically active barrier structures [7]. The excitation conditions in an STM are different to those in an SEM, but the present results show the capability of the STM-REBIC technique to characterize the cell structure observed in the surface of nc-Si films. In fact, it was not possible to get SEM-REBIC contrast from the cell boundaries in our samples due to the small dimensions of the cells which are below the resolution limit in the SEM-REBIC technique, while clear STM-REBIC contrast is achieved. The contrast obtained follows the peak and trough profile (see figure 1(d)) at the cell boundary which in the literature on conventional SEM-REBIC is explained by modelling the boundary as two Schottky barriers back to back [7]. The contrast obtained can be explained in terms of electrically active defects or regions which could be related to a higher accumulation of boron dopant at the cell boundaries. On the other hand, CITS images support this assumption through the differences in the local conductance measurements.

\section{Conclusions}

In summary, STM-REBIC enables us to resolve space-charge regions with an extension of tens of nanometres at the cell boundaries on nanocrystalline silicon films. Cell boundaries are electrically active for as-implanted layers with a moderate dose of dopant $\left(10^{15} \mathrm{~cm}^{-2}\right)$. In these samples, thermal treatment leads to a quenching of the internal built-in electric fields and no STM-REBIC contrast is detected. For a low implantation dose $\left(10^{13} \mathrm{~cm}^{-2}\right)$ the layers were too resistive to perform tunnelling experiments while after the annealing process some of the boundaries became electrically active as revealed by REBIC.

\section{Acknowledgments}

This work has been supported by MCYT (Project MAT 20002119). RP acknowledges MECD for the research grant SB2000-0164.

\section{References}

[1] Shimizu-Iwayama T, Nakao S and Saitoh K 1994 Appl. Phys. Lett. 651814

[2] Zhao X, Schoenfeld O, Kusano J, Aoyagi Y and Sugano T 1994 Japan. J. Appl. Phys. 33 L649

[3] Cullis A G, Canham L T and Calcott P D 1997 J. Appl. Phys. 82909

[4] Kanemitsu Y 1994 Phys. Rev. B 4916845

[5] Wolkin M V, Jorne J, Fauchet P M, Allan G and Delerue C 1999 Phys. Rev. Lett. 82197

[6] Holt D B 1994 Polycrystalline Semiconductors III-Physics and Technology, Solid State Phenomena vol 37-38 ed H P Strunk, J H Werner, B Fortin and O Bonnaud (Zurich: Trans. Tech.) p 171

[7] Palm J 1993 J. Appl. Phys. 741169

[8] Díaz-Guerra C and Piqueras J 1999 J. Appl. Phys. 861874

[9] Feenstra R M 1994 Surf. Sci. 299/300 965

[10] Hamers R J, Tromp R M and Demuth J E 1986 Phys. Rev. Lett. 561972

[11] Plugaru R, Craciun G, Nastase N, Méndez B, Cremades A, Piqueras J and Nogales E 2000 J. Porous Mater. 7291

[12] Nogales E, Méndez B, Piqueras J and Plugaru R 2001 Semicond. Sci. Technol. 16789

[13] Kazmerski L L 1991 J. Vac. Sci. Technol. B 91549

[14] Koschinski P, Kaufmann K and Balk L J 1994 Proc. 13th Int. Cong. on Electron Microscopy (Paris: Les Editions de Physique) p 1121 